\title{
The Effect of 17- $\beta$-estradiol on Cellular Proliferation Mediated by Protein Disulfide Isomerase and the Mammalian Target of Rapamycin (mTOR)
}

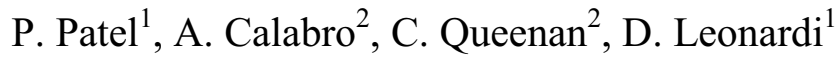 \\ 1. Bergen County Academies, Cell Biology Lab, 200 Hackensack Avenue, Hackensack, NJ 07601 \\ 2. Bergen County Academies, Nano-Structural Imaging Lab, 200 Hackensack Avenue, Hackensack, NJ \\ 07601
}

Protein disulfide isomerases (PDI) are a family of proteins localized in the endoplasmic reticulum that play a crucial role in protein folding [1]. Grp58/Erp27, a widely expressed protein in this family, has been found to play an important role in the production of mTOR, which promotes cell proliferation through the PI3K- mTOR pathway [2]. This pathway is regulated by two multi-protein complexes, mTORC1 and mTORC2 [2]. A recent study has shown that Grp58/Erp27 is involved in the assembly and folding of mTORC1 [2]. Additionally, 17- $\beta$-estradiol (E2) promotes the activation of the mTOR pathway. However, Grp58/Erp27 is inhibited by E2, which diminishes its enzymatic activity. As a result, excessive E2 causes accumulation of misfolded proteins in the ER, which can lead to apoptosis [3]. The goal of this study was to further evaluate the role of Grp58/Erp27 and mTOR in the presence of $\mathrm{E} 2$, in terms of cellular proliferation. It was hypothesized that exposure to increasing E2 concentrations, would cause the concentration of functional Grp58/Erp27 to diminish, and mTOR levels to decrease in the cell. As a result, cellular proliferation will decrease, and apoptosis may be promoted.

In this study, triple-negative human mammary gland adenocarcinoma (MDA-MB-231) cells were treated with $0.01 \mu \mathrm{M}, 0.1 \mu \mathrm{M}, 1 \mu \mathrm{M}, 10 \mu \mathrm{M}$ and $100 \mu \mathrm{M}$ E2, diluted in DMSO. An MTS assay showed that increasing concentrations of E2 were cytotoxic and decreased viability in a dose-response fashion. The decrease in cell viability at higher concentrations was statically significant $(\mathrm{p}<0.05)$. Additionally, an ELISA for PDI showed that with increasing E2 concentrations, the concentrations of PDI increased $(p<0.05)$ in a dose-response fashion. An ELISA for phosphorylated mTOR revealed that there was an overall decrease in phosphorylated mTOR as the concentrations of E2 increased. A caspase 3/7 assay and caspase 9 assay showed a statistically significant increase $(\mathrm{p}<0.05)$ of caspase $3 / 7$ for $100 \mu \mathrm{M} \mathrm{E} 2$, which clearly suggests that apoptosis, rather than necrosis, occurred in cells. Furthermore, a statistically significant increase $(\mathrm{p}<0.05)$ of caspase 9 for $100 \mu \mathrm{M}$ E2 implies that the apoptotic signal was induced internally. Light microscopy was used to evaluate cell morphology before and after treatment. At 100 $\mu \mathrm{M}$ E2, cells had an abnormal shape and granules were present outside of the cells, suggesting that the cells were stressed and trying to remove the E2 that was taken up (Figure 1).

These results show that high doses of E2 down-regulate mTOR, and that this process is mediated by PDI, suggesting that PDI should be further studied to understand its role in developing cancer.

References:

[1] T. Hashida et al., The Journal of Toxicological Sciences 36(1) (2011) 1-7

[2] I. Ramirez-Rangel et al., Molecular Cell Biology 31(8) (2011) 1657-1671

[3] Y. Honjo et al., Brain Research 1349 (2010) 90-96

[4] The authors would like to acknowledge the administration of the Bergen County Technical Schools for their continued support of the research program. 

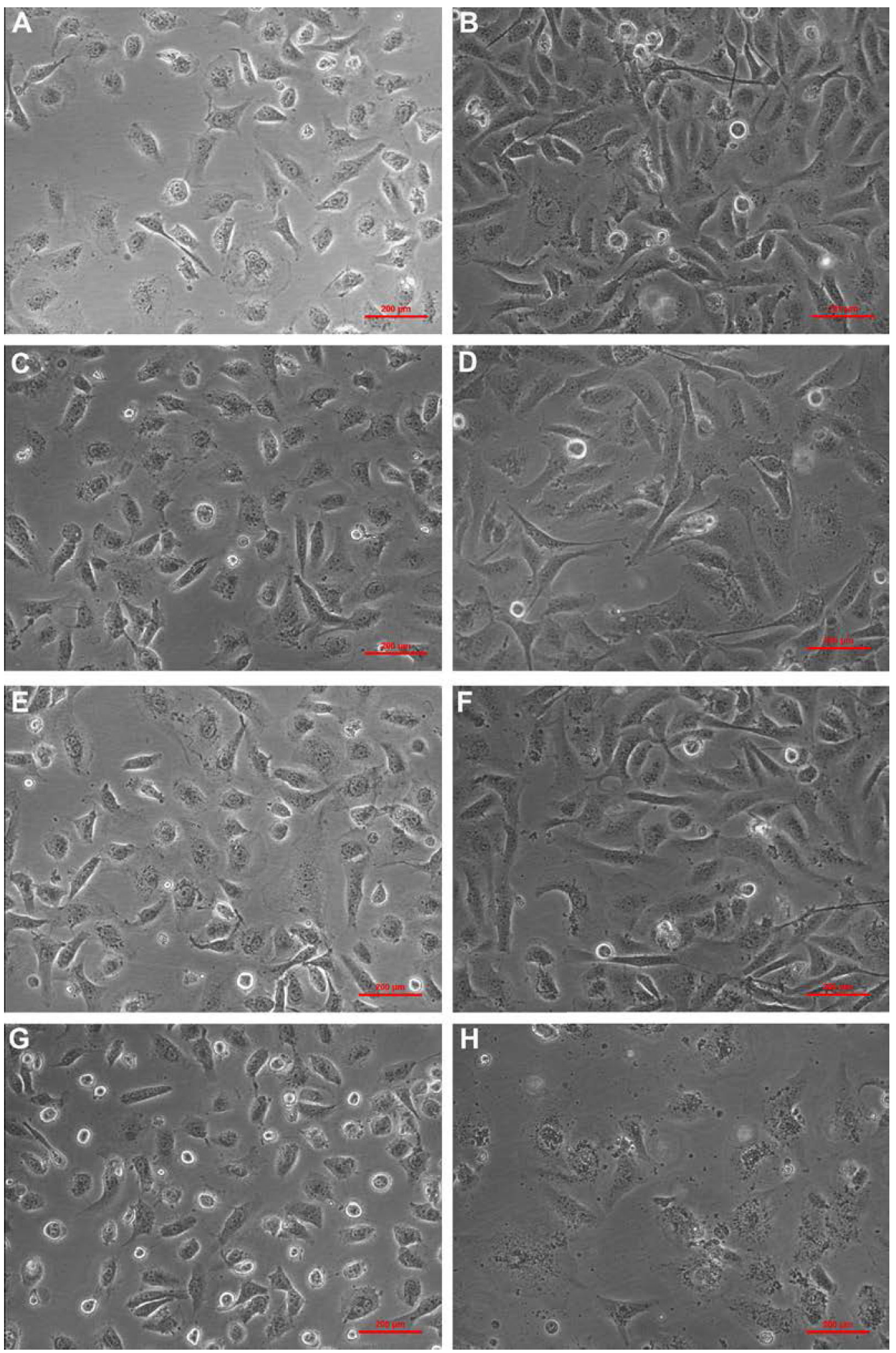

Figure 1: Light microscopy images of MDA-MB-231 cells treated with (A, B) DMSO, (C, D) $0.01 \mathrm{uM}$ E2, (D, E) $1.0 \mathrm{uM} \mathrm{E2,} \mathrm{(F,} \mathrm{G)} 100 \mathrm{uM} \mathrm{E2}$ at time zero (left) and 48 hours (right). Scale $=200 \mu \mathrm{m}$. 